\begin{tabular}{|c|l|}
\hline Title & Dielectric study of acetophenone and its derivatives \\
\hline Author(s) & Yomogida, Yoshiki; Nozaki, Ryusuke \\
\hline Citation & $\begin{array}{l}\text { Journal of Molecular Liquids, 149(3), 101-104 } \\
\text { https://doi.org/10.1016/.molliq.2009.09.001 }\end{array}$ \\
\hline Issue Date & 2009-10-30 \\
\hline Doc URL & http://hdl.handle.net/2115/39955 \\
\hline Type & article(author version) \\
\hline File Information & JML149-3_101.pdf \\
\hline
\end{tabular}

Instructions for use 


\title{
Dielectric study of acetophenone and its derivatives
}

\author{
Yoshiki Yomogida and Ryusuke Nozaki \\ Department of Physics, Faculty of Science, Hokkaido University, Sapporo, Hokkaido 060-0810, \\ Japan
}

\begin{abstract}
Complex permittivity measurements on acetophenone and its derivatives o-hydroxybenzaldehyde, o-methylacetophenone, and o-hydroxyacetophenone are performed at frequencies between $1 \mathrm{MHz}$ and $20 \mathrm{GHz}$ at temperatures from 273 to $323 \mathrm{~K}$. The parameters obtained from the fitting of the complex permittivity are analyzed in order to study the effects of the hydroxyl group within a molecule on the dielectric relaxation phenomenon in these liquids. The analysis indicates that dynamical properties are affected not only by the intermolecular hydrogen bond but also by the slight change in molecular structure. This conclusion differs from those obtained from the results of other experiments.
\end{abstract}

\section{Introduction}

Dielectric spectroscopy, which is used to measure the response of the complex permittivity to time-dependent electric fields, has contributed to our understanding of the molecular dynamics in the liquid state. With the development seen in experimental equipments, this method can now cover a very wide experimental frequency range [1] from $10 \mu \mathrm{Hz}$ to several $\mathrm{THz}$, the widest among the 
many spectroscopic methods. This experimental technique enables us to detect not only the short-time responses that depend on the dynamics of a single molecule or particular parts of a molecule (inter- and intramolecular vibrations), but also the long-time slow responses associated with the collective dynamics of some molecules (relaxation phenomenon). However, even with these capabilities, identification of the microscopic origin of a dielectric relaxation process is difficult because the relaxation process reflects the fluctuation of electric polarization which is a macroscopic phenomenon.

Among all liquids, the dielectric relaxation phenomenon in hydrogen-bonded liquids is the most peculiar and difficult to understand. In monohydric alcohols, which are basic hydrogen-bonded liquids, a dominant Debye-type relaxation process is observed in the lower-frequency range. Although this process has been observed for a long time by many researchers, the process remains a matter of interest and debate [2-6]. The peculiarity of the dielectric relaxation process in these liquids is often attributed to the presence of hydrogen bonds. However, the molecular origin of the process is not generally agreed upon, and it is necessary to accumulate systematic research to understand how the formation of hydrogen bonds affects the macroscopic relaxation phenomenon.

In this paper, we report the results of complex permittivity measurements on acetophenone (AP) and its derivatives o-hydroxybenzaldehyde (OHBA), o-methylacetophenone (OMAP), and o-hydroxyacetophenone (OHAP). These chemicals are convenient for a systematic study of dielectric relaxation because they differ only in their side chains and number of hydroxyl groups; nevertheless few papers have been published on the dielectric relaxation of AP derivatives [7]. OHBA and OHAP 
possess a hydroxyl group and form intermolecular hydrogen bonds, while AP and OMAP do not have hydroxyl groups.

The main objective of this study is to investigate the influence of the presence of a hydroxyl group on the dielectric relaxation phenomenon. Moreover, we compare the results of the dielectric measurements with those of the viscosity measurements and optical Kerr effect (OKE) [8] reported by Wang. Using the comparisons of data from the dielectric measurements and from other techniques, we discuss the relationship between the dielectric relaxation phenomenon and the dynamics at a molecular level for hydrogen-bonded liquids.

\section{Experiment}

To cover frequencies between $1 \mathrm{MHz}$ and $20 \mathrm{GHz}$, two different experimental setups were used.

For the 1 to $500 \mathrm{MHz}$ range, the complex permittivity measurements were performed with a network analyzer (HP4195A). The coaxial sample cell was composed of an outer conductor with an inner diameter of $3.5 \mathrm{~mm}$ and a center conductor with an outer diameter of $2 \mathrm{~mm}$. The length of the outer conductor was $10 \mathrm{~mm}$. The values of the complex permittivity were obtained from reflection measurements with the sample cell located at the end of a coaxial line. For the $500 \mathrm{MHz}$ to $20 \mathrm{GHz}$ range, time domain reflectometry $[9,10]$ was employed. A flat-end capacitor cell using a 2 -mm semi-rigid coaxial waveguide was used in this measurement. The temperature of the sample cells in both experimental setups was maintained at temperatures between 273 and $323 \mathrm{~K}$ with an accuracy of $0.1 \mathrm{~K}$. 
Viscosity measurements were taken using Canon-Fenske viscometers at $298 \mathrm{~K}$.

The chemicals AP, OHBA, OMAP, and OHAP were obtained from Kanto Chemical and used without further purification. The molecular structures are shown in Fig. 1 and the molecular weights of AP, OHBA, OMAP, and OHAP are 120.15, 122.12, 134.18, and $136.15 \mathrm{~kg} / \mathrm{mol}$, respectively.

3. Results and discussion

Figures 2(a)-(d) show the complex permittivities, $\varepsilon^{*}(\omega)=\varepsilon^{\prime}(\omega)-i \varepsilon^{\prime \prime}(\omega)$, of AP, OHBA, OMAP, and OHAP at $298 \mathrm{~K}$, respectively. The complex permittivities of AP and OHBA can be described by the Cole-Cole function [11] in Eq. (1), while the complex permittivities of OMAP and OHAP can be described by the Davidson-Cole function [12] in Eq. (2).

$$
\begin{aligned}
& \varepsilon^{*}(\omega)=\frac{\Delta \varepsilon}{1+(i \omega \tau)^{\delta}} \\
& \varepsilon^{*}(\omega)=\frac{\Delta \varepsilon}{\left(1+i \omega \tau_{m}\right)^{\gamma}}
\end{aligned}
$$

Several parameters were derived from the fitting of Eqs. (1) and (2) to the complex permittivities obtained and shown in Figs. 3, 4, and 5 which are the temperature dependence plots of the relaxation frequency, relaxation strength, and shape parameter, respectively. These parameters change smoothly with the temperature.

Fröhlich theory [13] expresses the static permittivity $\mathcal{E}_{S}$ as follows:

$$
\varepsilon_{s}-\varepsilon_{\infty}=\frac{3 \varepsilon_{s}}{2 \varepsilon_{s}+\varepsilon_{\infty}}\left(\frac{\varepsilon_{\infty}+2}{3}\right)^{2} \frac{N_{A} c g \mu^{2}}{3 \varepsilon_{0} k_{B} T} .
$$


Here, $\varepsilon_{\infty}$ is the high-frequency permittivity, $N_{A}$ is Avogadro's number, $k_{B}$ is the Boltzmann constant, $c$ is the concentration of the dipole molecules, $\mu$ is the electric dipole moment in the gaseous state, $g$ is the dipole-dipole correlation factor, and $\varepsilon_{0}$ is the vacuum permittivity. The dipole-dipole correlation factor can be calculated using Eq. (3). The values $3.12 \mathrm{D}$ for AP, 2.94 D for OHBA, 2.83 D for OMAP, and 3.54 D for OHAP are employed [14] as electric dipole moments $\mu$ and $\varepsilon_{\infty}$ is set equal to $n^{2}$, where $n$ is the refractive index. Although the values of $g$ obtained in the calculation are not very accurate, we can obtain the relative values and discuss their tendencies.

Figure 6 shows the dependence of the $g$ factor on the viscosity at $298 \mathrm{~K}$. The $g$ factor is the index of the local order of dipoles in liquids. If there is no correlation between dipoles, the $g$ factor is equal to 1 . If there is the effect of a dipole to orient the surrounding dipoles in the parallel direction, $g>1$, and in the anti-parallel direction, $g<1$. The $g$-factor usually reflects the molecular structure and forces of the local type. In the case of AP derivatives studied here, the local type forces (intermolecular interaction) mainly contribute to the change in the $g$-factor because the molecular structures of AP derivatives are almost the same.

Figure 6 shows that the $g$-factor is in the order AP $<$ OHBA $<$ OMAP $<$ OHAP. The $g$-factor of OHBA is much smaller than that of OHAP, though both are considered to form an intermolecular interaction in the same way. And the $g$ factor of OHBA is even smaller than that of OMAP which does not have a hydroxyl group. On the other hand, the viscosity is in the order $\mathrm{AP}<\mathrm{OMAP}<$ OHBA $<$ OHAP. The viscosities of OHBA and OHAP are much higher than those of AP and OMAP, and this is due to the formation of intermolecular hydrogen bonds. Judging by the results of the 
viscosity measurements, it is considered that the presence of a hydroxyl group in a molecule is the main factor for determining the dynamical properties and OHBA and OHAP have the same manner of intermolecular hydrogen bonding. On the contrary, the comparison of the $g$-factors in this work indicates that the manner of the correlation between dipoles in OHBA and OHAP differ slightly. In the following sections, the influence of the difference on the dynamical properties is discussed.

According to Eyring's theory of rate process, the relaxation frequency $f_{m}=\left(2 \pi \tau_{m}\right)^{-1}$ is given by

$$
\log f_{m}=\log f_{0}+\frac{\Delta E}{2.303 R T}
$$

Here, $R$ is the gas constant, $T$ is the absolute temperature, $\Delta E$ is the activation energy associated with the dipole orientation, and $f_{0}$ represents the extrapolated frequency at $T \rightarrow \infty$. The activation energies of AP derivatives are determined by Eq. (2) and the values are plotted against viscosity in Fig. 7. The activation energy is not proportional to the viscosity as well as the $g$ factor. The results indicate that the correlation between dipoles affect the response of the dipolar reorientation to an applied electric field.

Wang et al. [8] reported the result of the OKE of AP derivatives at $298 \mathrm{~K}$ to investigate the molecular relaxation. In the case of the OKE, the relaxation time was completely proportional to the macroscopic viscosity. This proportional relationship indicated that molecular dynamics of AP derivatives was a simple reorientation of a molecule, and an intermolecular hydrogen bond increased the macroscopic viscosity. On the other hand, as seen in Fig. 8, the relationship of the dielectric relaxation time $\tau_{m}$ with the viscosity differs from the OKE measurements. We did not get 
a reasonable explanation for the inconsistency of the behavior of the macroscopic dielectric relaxation and the molecular relaxation of a molecule that was observed in the OKE measurements. Nevertheless, on the basis of the dielectric measurements, it is clear that the existence of the hydroxyl group in a molecule is not the only factor controlling the electric polarization.

In our work, we expected the intermolecular interaction to be the dominant factor to the $g$ factor and the dynamical properties $\left(\Delta E\right.$ and $\left.\tau_{m}\right)$ because the molecular structures of $\mathrm{AP}$ derivatives are almost the same. As explained, however, molecular structures may affect the dielectric properties as well as the interaction. Future investigations on AP derivatives are necessary to see what controls the correlation between molecules in these liquids.

In Fig. 5, the shape parameters of OMAP and OHAP that both exhibit larger $g$-factor are almost equal to 1 and slightly dependent on temperature, whereas the parameters are greatly shifted from 1 and depend on the temperature in the case of AP and OHBA. These results again indicate that the existence of a hydrogen bond is not the only determining factor in explaining the dielectric relaxation in these liquids.

\section{Conclusion}

In the present study, comprehensive complex permittivity measurements were carried out on AP derivatives to investigate the influence of the hydrogen bond on dielectric relaxation and molecular dynamics in hydrogen-bonded liquids. The dipole-dipole correlation factor $g$ was estimated from the analysis of the static permittivity of several samples. The $g$ factor of OHBA is 
much smaller than that of OHAP, though both possess a hydroxyl group and are expected to form intermolecular hydrogen bonds. Surprisingly, the $g$-factor of OHBA is even smaller than that of OMAP which does not have a hydroxyl group. The results indicate that the presence of a hydrogen bond cannot fully explain the correlation between dipoles in AP derivatives. Furthermore, the correlation between dipoles also affects the dynamical properties observed in the relaxation time $\tau_{m}$ and the activation energy $\Delta E$.

The viscosities of OHBA and OHAP are larger than those of AP and OMAP. With the results of the viscosities, the molecular motion in AP derivatives can be simply classified by the existence of a hydroxyl group in a molecule. This conclusion is also supported by the results of OKE measurements [8]. Thus, the pictures of the molecular dynamics in AP derivatives obtained from dielectric measurements and other measurements are totally different. We need to proceed with studies of these liquids in order to clarify this difference. Nevertheless, this research provides us with a considerable amount of information on the dielectric relaxation phenomenon in hydrogen-bonded liquids.

\section{References}

[1] F. Kremer, A. Schönhals, Broadband Dielectric Spectroscopy, Springer, Berlin, 2003.

[2] L. M. Wang, R. Richer, J. Chem. Phys. 121 (2004) 11170.

[3] L. M. Wang, R. Richert, J. Phys. Chem. B 109 (2005) 11091.

[4] L. M. Wang, S. Shahriari, R. Richert, J. Phys. Chem. B 109 (2005) 23255. 
[5] T. El Goresy, R. Böhmer, J. Chem. Phys. 128 (2008) 154520.

[6] B. Jakobsen, C. Maggi, T. Christensen, J. C. Dyre, J. Chem. Phys. 129 (2008) 184502.

[7] S. L. A. Messieh, J. Mol. Liq. 105 (2003) 37.

[8] Y. Wang, K. Ushida, Y. Tominaga, A. Kira, Chem. Phys. Lett. 299 (1999) 576.

[9] R. Nozaki, T. K. Bose, IEEE Trans. Instrum. Meas. 39 (1990) 945.

[10] R. Nozaki, Solid State Phys. (Tokyo) 28 (1993) 505.

[11] K. S. Cole, R. H. Cole, J. Chem. Phys. 9 (1941) 341.

[12] D. W. Davidson, R. H. Cole, J. Chem. Phys. 18 (1951) 1417.

[13] H. Fröhlich, Theory of Dielectrics, Claredon, Oxford, 1958.

[14] A. L. McClellan, Tables of Experimental Dipole Moments, W. H. FREEMAN AND COMPANY, San Francisco, 1963.

Figure captions

Fig. 1. The molecular structure of (a) acetophenone (AP), (b) o-hydroxybenzaldehyde (OHBA), (c) o-methylacetophenone (OMAP), and (d) o-hydroxyacetophenone (OHAP).

Fig. 2. Complex permittivity of (a) acetophenone (AP), (b) o-hydroxybenzaldehyde (OHBA), (c) o-methylacetophenone (OMAP), and (d) o-hydroxyacetophenone (OHAP) at $298 \mathrm{~K}$. Experimental results are shown in solid squares (๑). For 2(a) and 2(c), the solid line is from the fitting of Eq. (1). For 2(b) and 2(d), the solid line is from the fitting of Eq. (2). 
Fig. 3. Arrhenius diagram of acetophenone derivatives. The relaxation frequency $f_{m}$ is calculated from dielectric relaxation time $\tau_{m}$ with the equation $f_{m}=\left(2 \pi \tau_{m}\right)^{-1}$.

Fig. 4. Temperature dependence of relaxation strength $\Delta \varepsilon$ for acetophenone derivatives.

Fig. 5. Temperature dependence of symmetric shape parameter $\delta$ in Eq. (1) for AP and OHBA and asymmetric parameter $\gamma$ in Eq. (2) for OMAP and OHAP.

Fig. 6. Dipole-dipole correlation factor $g$ plotted against viscosity at $298 \mathrm{~K}$. The $g$-factor is calculated from the static permittivities of acetophenone derivatives with Eq. (3).

Fig. 7. Activation energy $\Delta E$ plotted against viscosity at $298 \mathrm{~K}$.

Fig. 8. Dielectric relaxation time $\tau_{m}$ plotted against viscosity at $298 \mathrm{~K}$. 

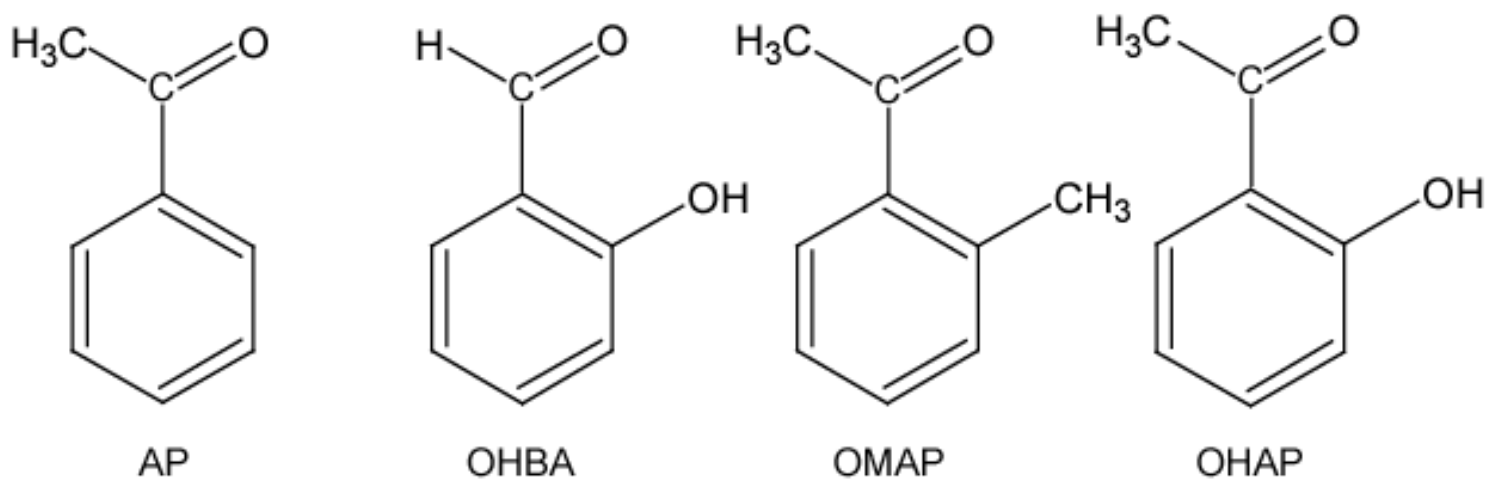

Fig.1

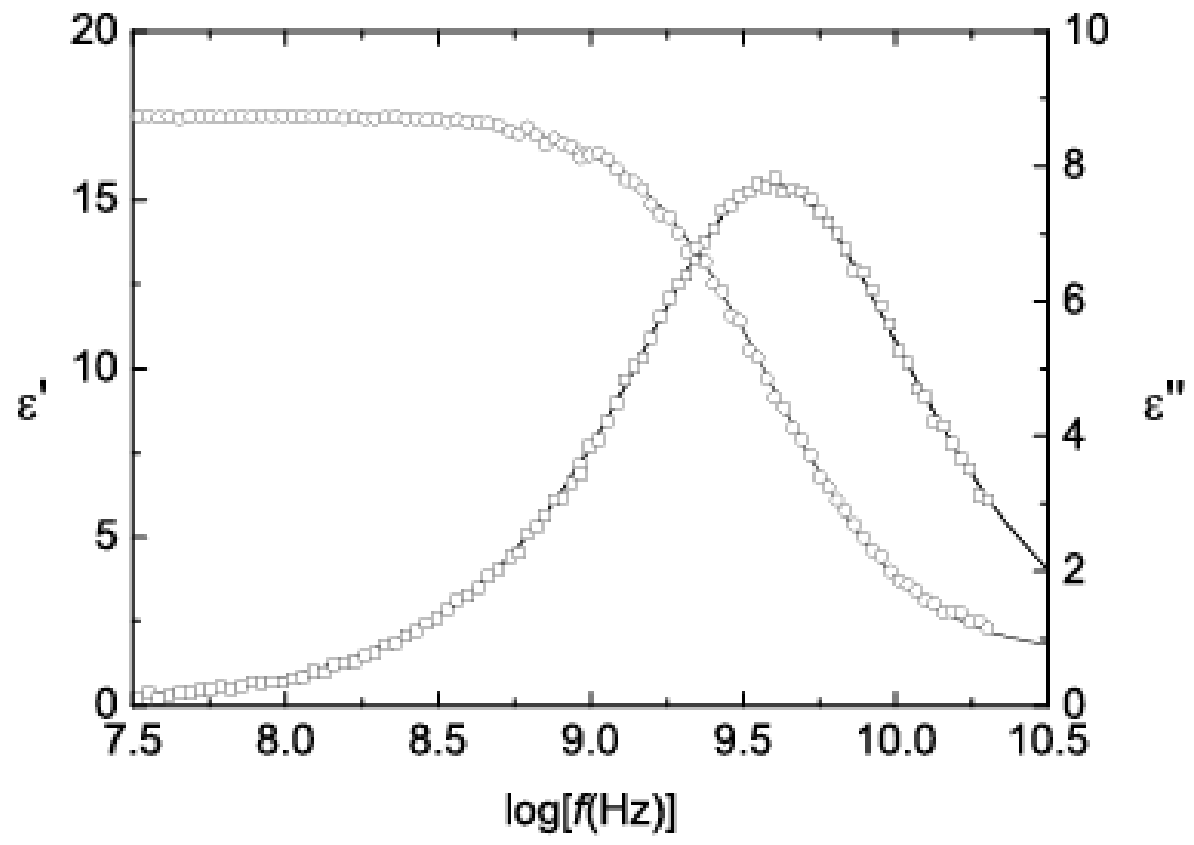

Fig.2(a)

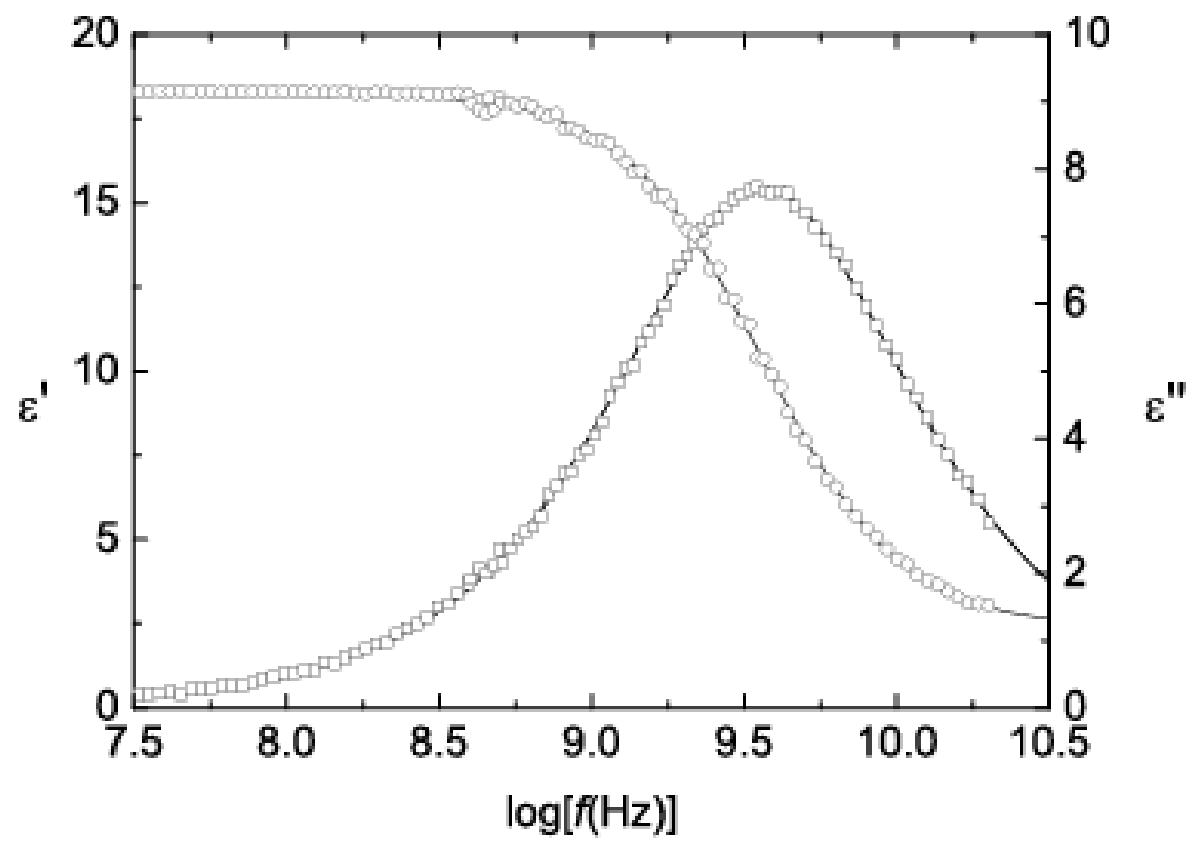

Fig.2(b) 


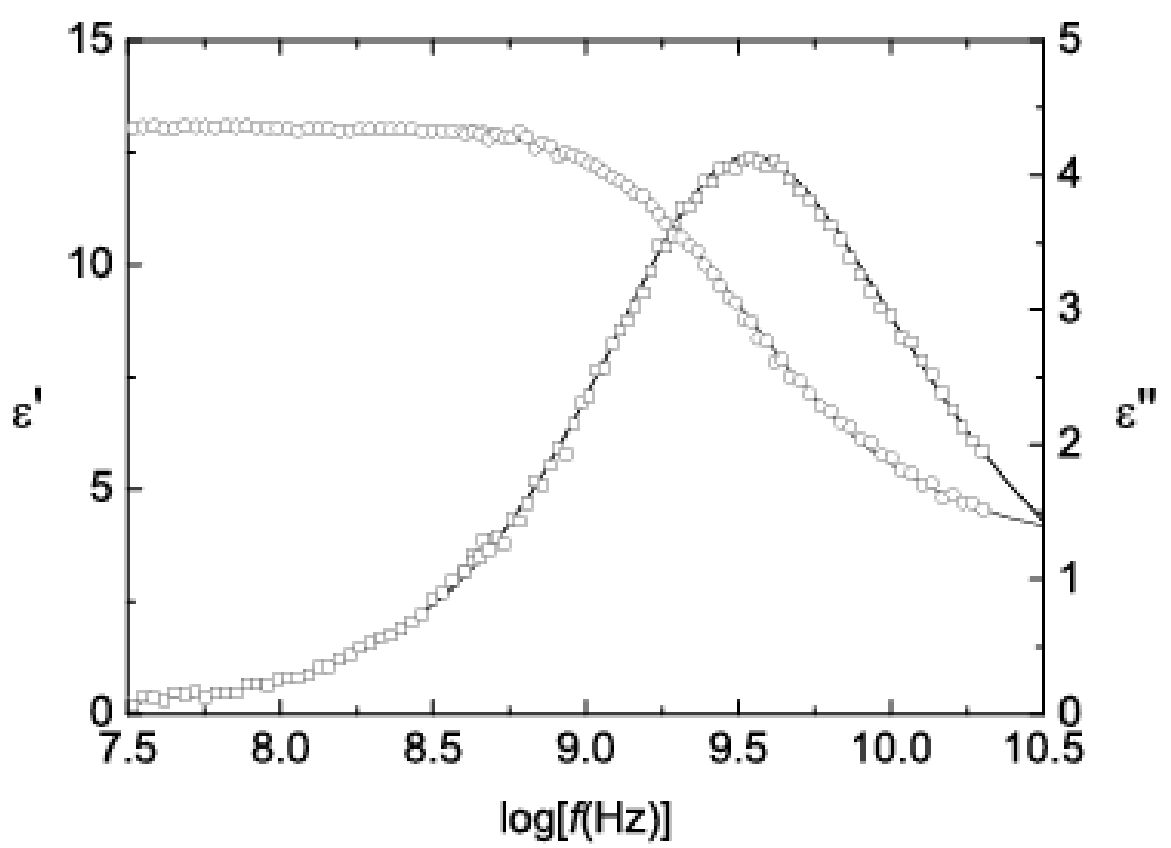

Fig.2(c)

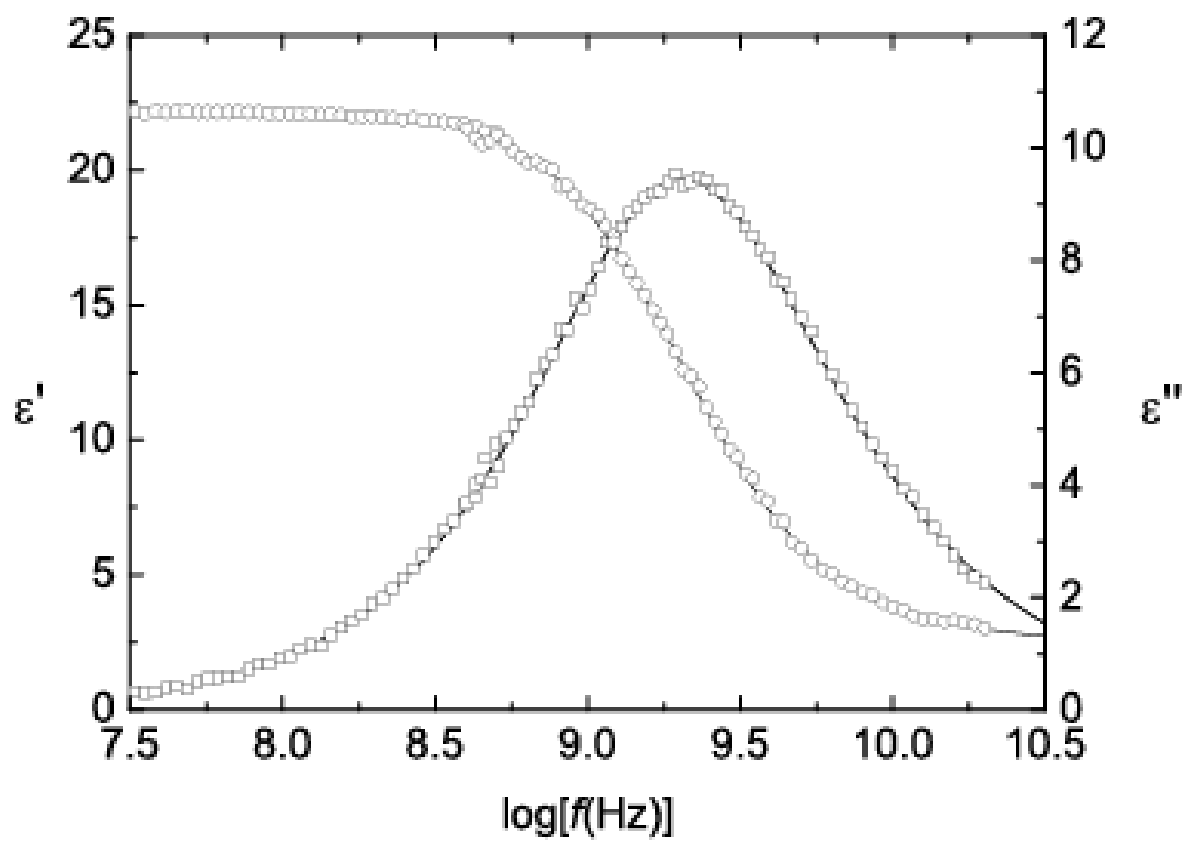

Fig.2(d) 


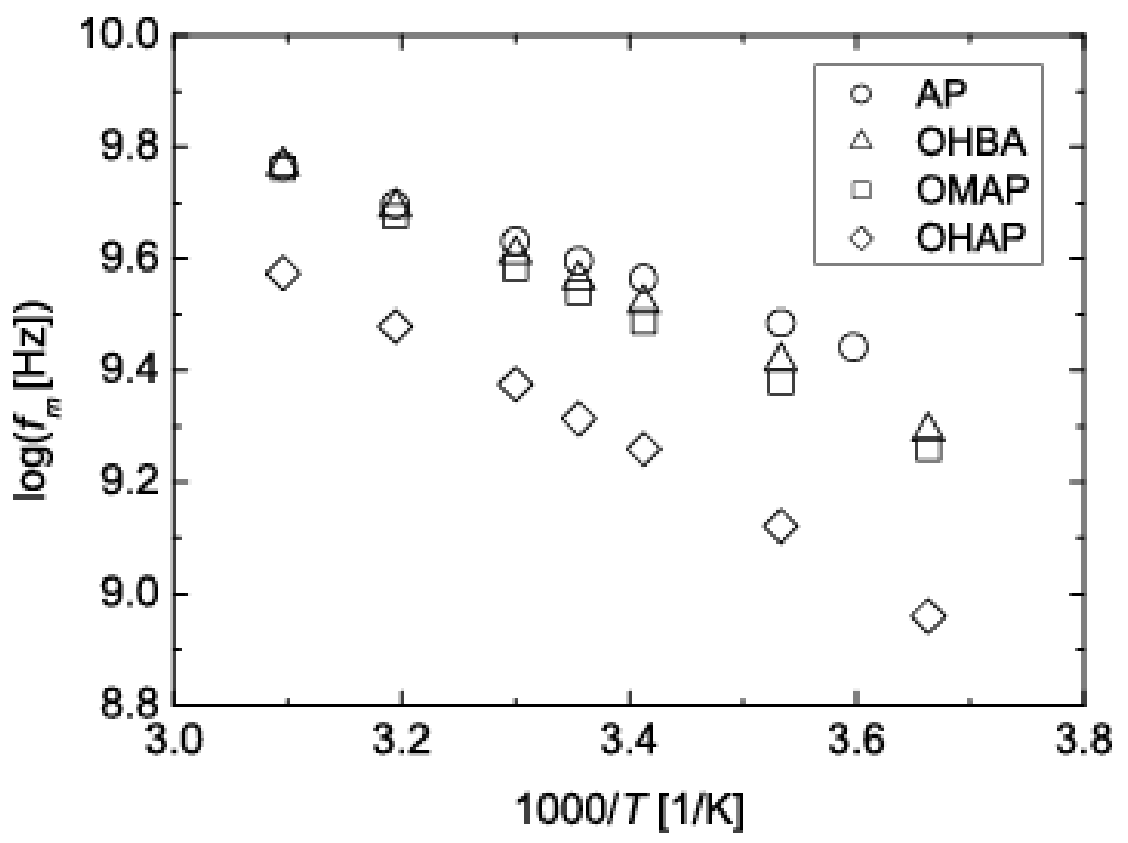

Fig. 3

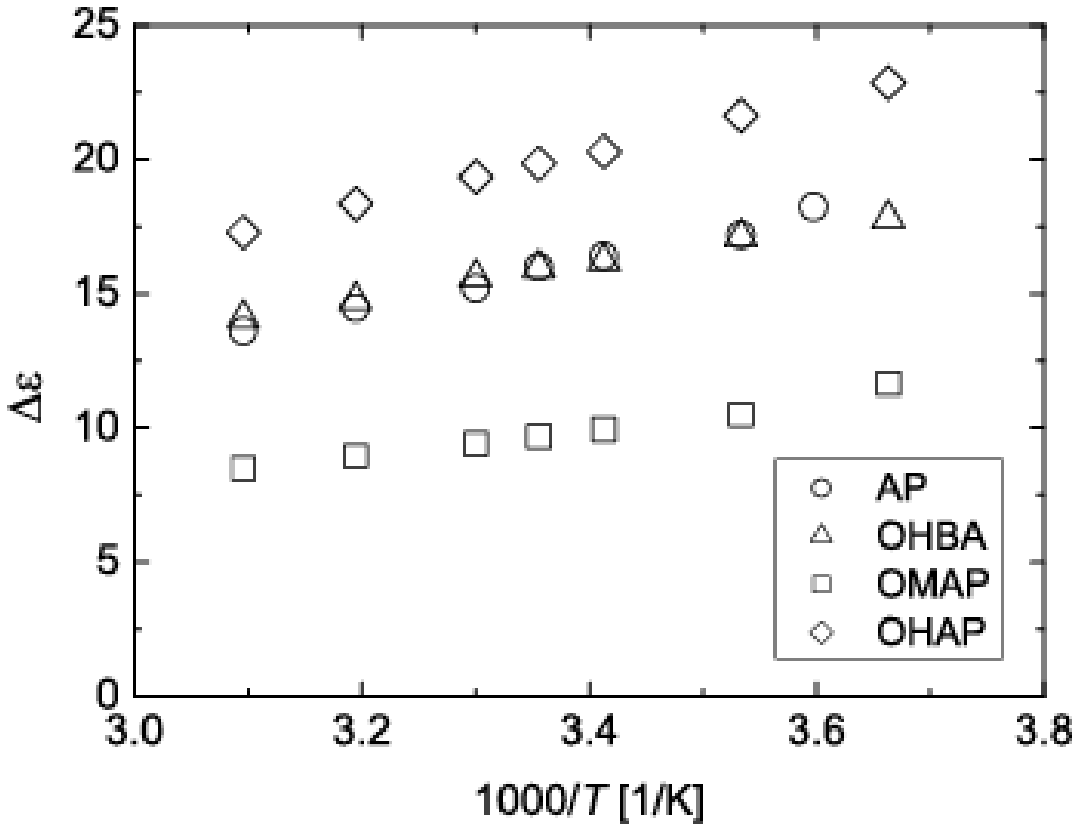

Fig. 4 


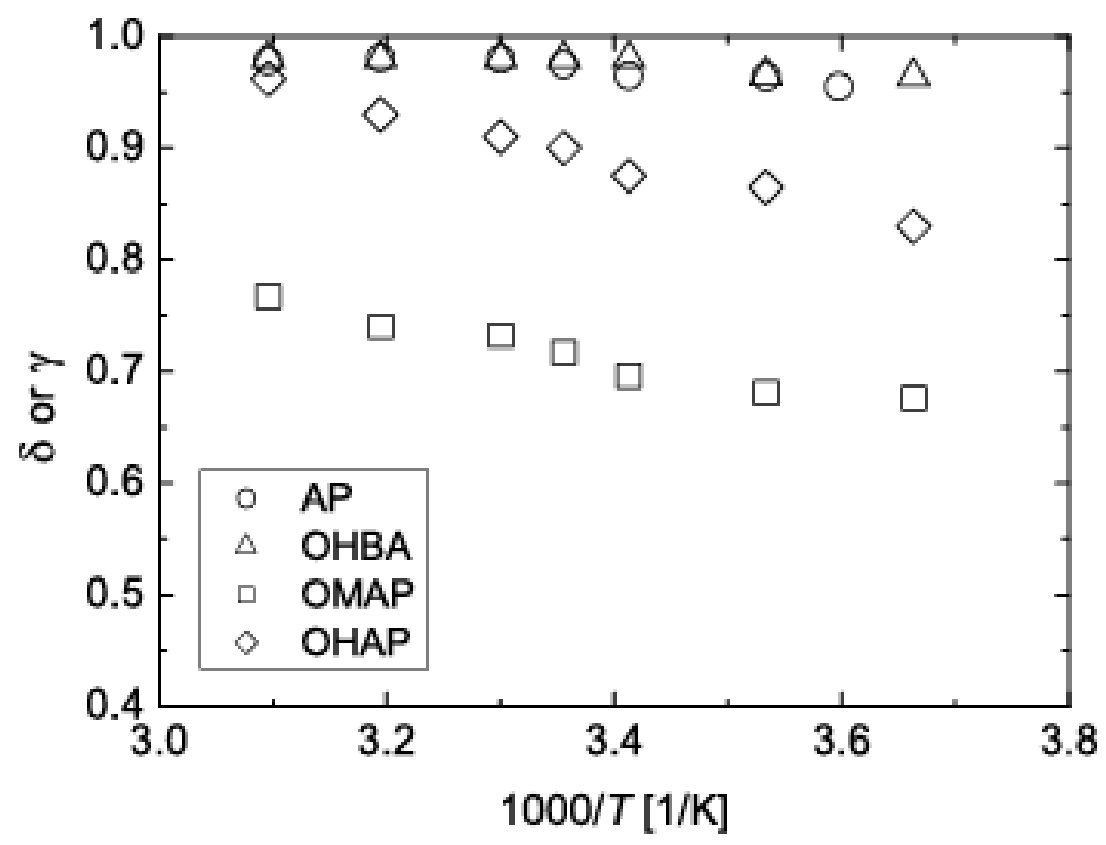

Fig.5

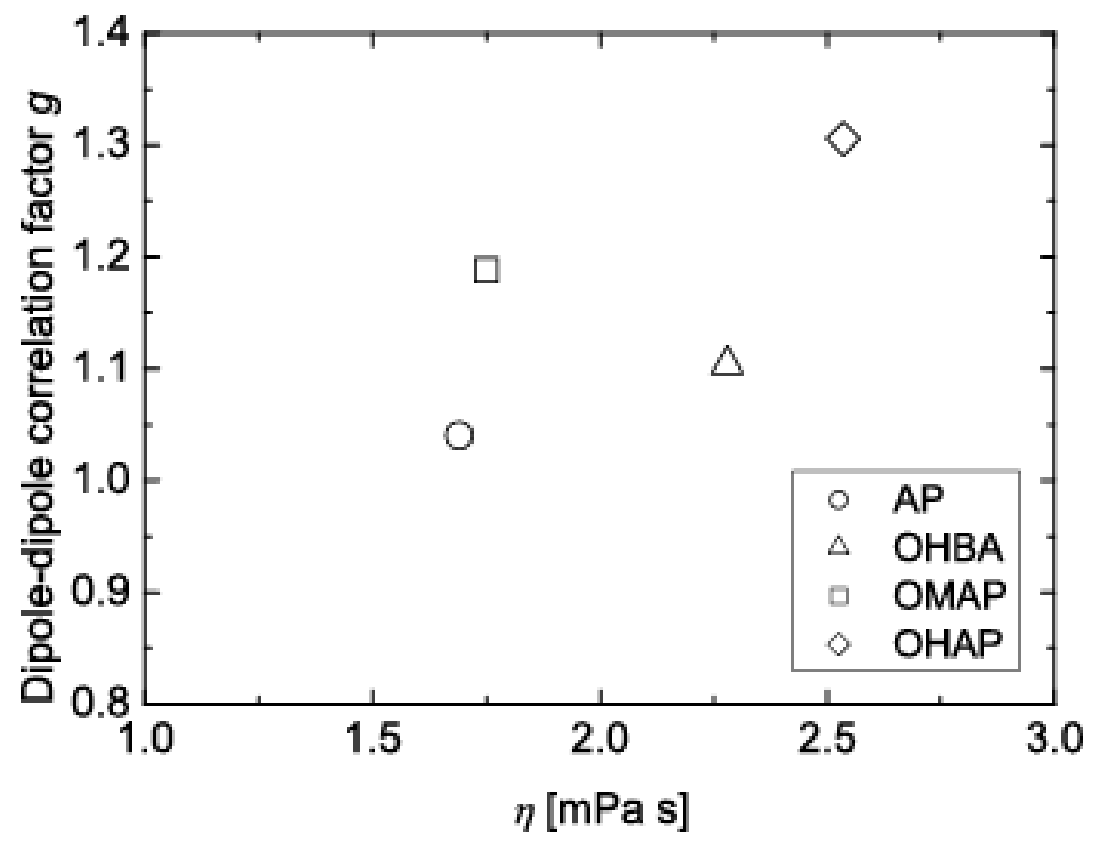

Fig.6 


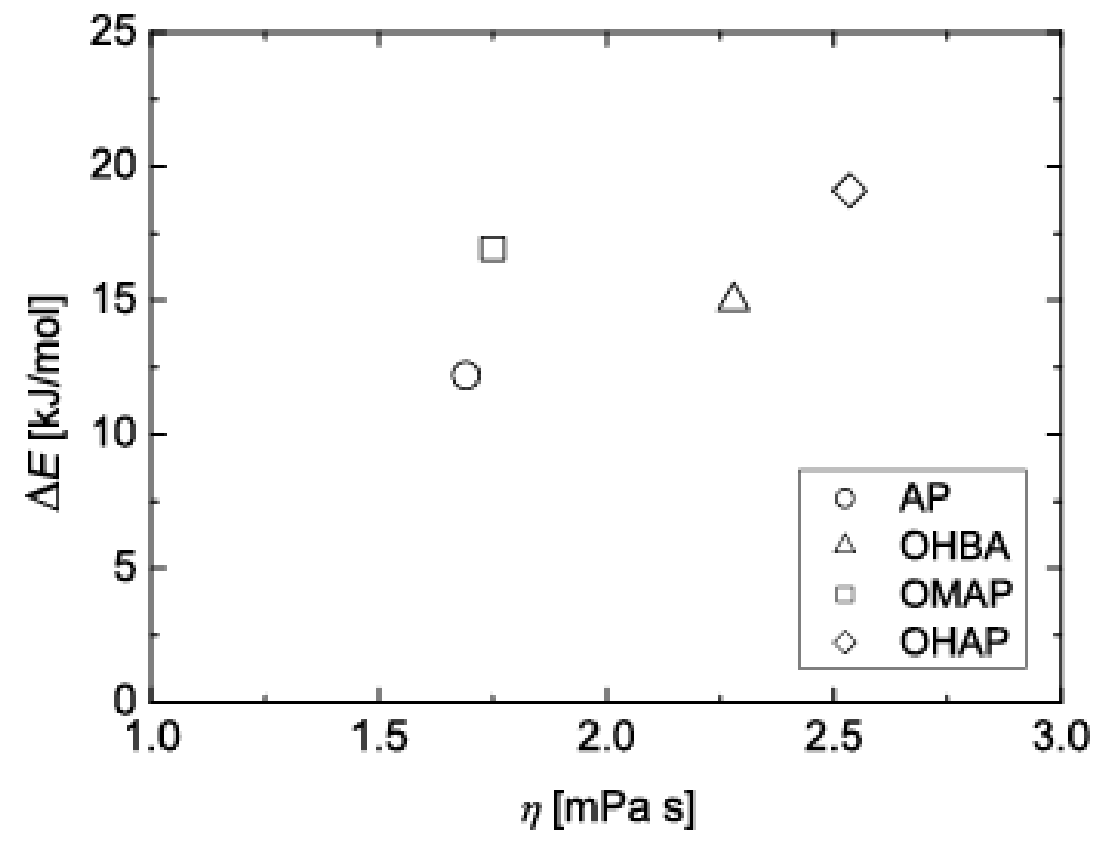

Fig. 7

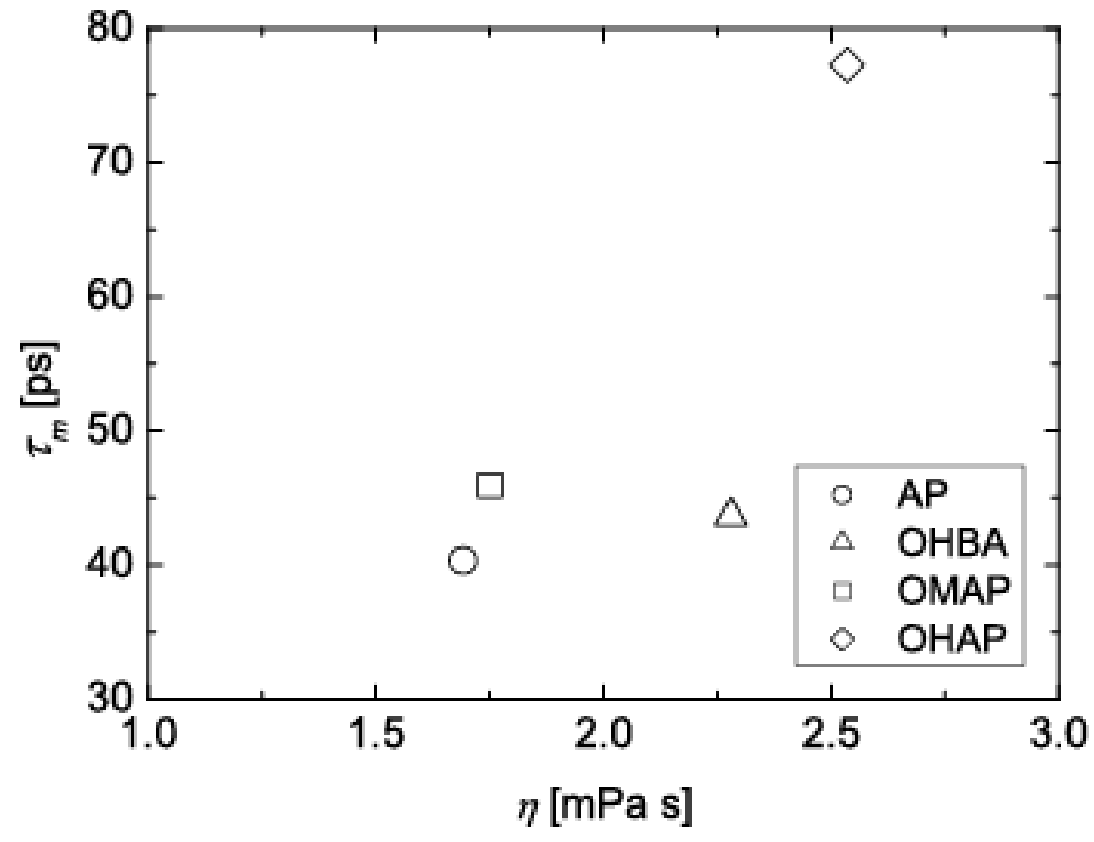

Fig. 8 